\title{
Molecular characterization of the stomach microbiota in patients with gastric cancer and in controls
}

\author{
Correspondence \\ Lars Engstrand \\ lars.engstrand@smi.ki.se
}

Received 13 October 2008

Accepted 11 December 2008

\author{
Johan Dicksved, ${ }^{1}$ Mathilda Lindberg, ${ }^{2,3}$ Magnus Rosenquist, ${ }^{4}$ \\ Helena Enroth, ${ }^{5}$ Janet K. Jansson ${ }^{1,6}$ and Lars Engstrand ${ }^{2,3}$
}

\author{
${ }^{1}$ Department of Microbiology, Swedish University of Agricultural Sciences, Uppsala, Sweden \\ ${ }^{2}$ Swedish Institute for Infectious Disease Control, 17182 Solna, Sweden \\ ${ }^{3}$ Department of Microbiology, Tumor and Cell Biology, Karolinska Institutet, 17177 Stockholm, \\ Sweden \\ ${ }^{4}$ Department of Oncology, Radiology and Clinical Immunology, Uppsala University Hospital, 75185 \\ Uppsala, Sweden \\ ${ }^{5}$ Department of Clinical Microbiology, Unilabs AB, Kärnsjukhuset, Skövde, Sweden \\ ${ }^{6}$ Lawrence Berkeley National Laboratory, Division of Earth Sciences, Berkeley, CA, USA
}

Persistent infection of the gastric mucosa by Helicobacter pylori can initiate an inflammatory cascade that progresses into atrophic gastritis, a condition associated with reduced capacity for secretion of gastric acid and an increased risk of developing gastric cancer. The role of $H$. pylori as an initiator of inflammation is evident but the mechanism for development into gastric cancer has not yet been proven. A reduced capacity for gastric acid secretion allows survival and proliferation of other microbes that normally are killed by the acidic environment. It has been postulated that some of these species may be involved in the development of gastric cancer; however, their identities are poorly defined. In this study, the gastric microbiota from ten patients with gastric cancer was characterized and compared with that from five dyspeptic controls using the molecular profiling approach terminal restriction fragment length polymorphism (T-RFLP), in combination with $16 \mathrm{~S}$ rRNA gene cloning and sequencing. T-RFLP analysis revealed a complex bacterial community in the cancer patients that was not significantly different from that in the controls. Sequencing of 140 clones revealed 102 phylotypes, with representatives from five bacterial phyla (Firmicutes, Bacteroidetes, Proteobacteria, Actinobacteria and Fusobacteria). The data revealed a relatively low abundance of $H$. pylori and showed that the gastric cancer microbiota was instead dominated by different species of the genera Streptococcus,

Lactobacillus, Veillonella and Prevotella. The respective role of these species in development of gastric cancer remains to be determined.

\section{INTRODUCTION}

Gastric cancer is a global health problem, with the highest rates in developing countries. Research during the past few decades has shown that Helicobacter pylori is the major causative agent in gastric cancer, although the aetiological mechanism is not yet fully understood. The majority of the individuals that are infected by $H$. pylori develop mild pangastritis, where the gastric physiology is maintained and not associated with disease (Smith et al., 2006). However,

Abbreviations: T-RFLP, terminal restriction fragment length polymorphism; TRF, terminal restriction fragment.

The GenBank/EMBL/DDBJ accession numbers for the unique sequences generated in this study are FJ268879-FJ268953.
H. pylori infection can also lead to severe diseases. In antral predominant gastritis, the production of gastric acid is increased (hyperchlorhydria), which is associated with a high risk of development of duodenal ulcer disease, but protective against gastric cancer development (Hansson et al., 1996). By contrast, corpus predominant gastritis leads to lower production of gastric acid (hypochlorhydria) and may lead to atrophic gastritis, a condition with increased risk of developing gastric cancer (Uemura et al., 2001). The hypochlorhydric condition creates an environment suitable for other micro-organisms that can enter and colonize the stomach. Some of these bacteria are nitrogen-reducing bacteria that are able to produce carcinogenic $N$-nitroso compounds through conversion of nitrates or nitrites from 
Table 1. Clinical characteristics for gastric cancer patients and dyspeptic controls

\begin{tabular}{|c|c|c|c|c|c|c|c|c|}
\hline \multirow[t]{2}{*}{ Sample ID } & \multirow[t]{2}{*}{ Age (years) } & \multirow[t]{2}{*}{ Gender } & \multirow[t]{2}{*}{ Group } & \multirow[t]{2}{*}{ H. pylori culture } & \multicolumn{3}{|c|}{ H. pylori serology } & \multirow[t]{2}{*}{ H. pylori total } \\
\hline & & & & & ELISA & imm & p116 & \\
\hline 1 & 88 & M & Cancer & 0 & 0 & 0 & 0 & 0 \\
\hline 2 & 52 & M & Cancer & 1 & 1 & 1 & 1 & 1 \\
\hline 3 & 58 & M & Cancer & 0 & 1 & 1 & 1 & 1 \\
\hline 4 & 66 & $\mathrm{~F}$ & Cancer & 0 & 1 & 0 & 0 & 1 \\
\hline 5 & 67 & M & Cancer & 0 & 1 & 1 & 1 & 1 \\
\hline 6 & 77 & M & Cancer & 0 & 1 & 1 & 1 & 1 \\
\hline 7 & 73 & M & Cancer & 1 & 1 & 1 & 1 & 1 \\
\hline 8 & 77 & M & Cancer & 0 & 1 & 1 & 1 & 1 \\
\hline 9 & 77 & $\mathrm{~F}$ & Cancer & 0 & 0 & 0 & 0 & 0 \\
\hline 10 & 72 & M & Cancer & 1 & $\mathrm{ND}^{*}$ & 1 & 1 & 1 \\
\hline C11 & 68 & M & Control & 0 & 0 & 0 & 0 & 0 \\
\hline $\mathrm{C} 12$ & 75 & M & Control & 0 & 0 & 0 & 0 & 0 \\
\hline C13 & 75 & M & Control & 0 & 0 & 0 & 0 & 0 \\
\hline C14 & 73 & $\mathrm{~F}$ & Control & 0 & 0 & 0 & 0 & 0 \\
\hline C15 & 75 & $\mathrm{~F}$ & Control & 0 & 0 & 0 & 0 & 0 \\
\hline
\end{tabular}

${ }^{\star} \mathrm{ND}$, Not determined.

the saliva (Ziebarth et al., 1997). Secretion of vitamin C to gastric juice by healthy mucosa inhibits the bacterial synthesis of nitrites to $\mathrm{N}$-nitroso compounds. However, in hypochlorohydric gastric juice, vitamin $\mathrm{C}$ levels are reduced (Schorah et al., 1991). Instead, the nitrite levels are increased and available for the bacteria that are present in the hypochlorhydric stomach (Mowat et al., 2000). This raises the question whether bacteria other than $H$. pylori contribute to development of cancer. In fact, $H$. pylori colonizes the atrophic stomach poorly, and in intestinal metaplasia hardly at all, suggesting that $H$. pylori creates an environment (atrophy and hypochlorhydria) suitable for gastric carcinogenesis rather than causing the cancer directly (Blaser \& Atherton, 2004).

It is known that the microbiota in individuals with gastric cancer or precancerous conditions is altered from the normal gastric microbiota. However, information on which species are present during these altered physiological states is scarce. The gastric microbiota has, however, been studied in hypochlorhydric conditions in subjects using acid-reducing drugs, but these studies have relied on cultivation approaches (Adamsson et al., 1999; Mowat et al., 2000). A large fraction of the microbes residing in the gut have not yet been cultivated (Bik et al., 2006; Eckburg et al., 2005), therefore suggesting a skewed representation from culture-based studies. Although the development of molecular tools has bypassed the necessity for cultivation, very few studies have investigated the microbial composition of the stomach using culture-independent approaches (Bik et al., 2006; Kato et al., 2006; Monstein et al., 2000; Andersson et al., 2008).

In the present study, terminal restriction fragment length polymorphism (T-RFLP) was used in combination with cloning and sequencing of $16 \mathrm{~S}$ rRNA genes to assess the composition of the gastric microbiota of subjects with gastric cancer. The aim was to describe the composition of the gastric microbiota in patients with gastric cancer and compare it with that in subjects with normal gastric mucosal morphology.

\section{METHODS}

Patient cohort. The clinical material in this study was derived from a hospital-based study described previously (Enroth et al., 2000). Stomach biopsies from ten patients with non-cardia gastric cancer (five with intestinal type and five with diffuse type) and five dyspeptic control patients with normal gastric mucosal morphology were included in this study. Biopsies derived from non-cancerous gastric mucosa of antrum and corpus were pooled together. All subjects were included after providing informed consent, and the study was approved by the ethics committee of the Medical Faculty, Uppsala University DNR UU96/95 (Uppsala, Sweden). None of the individuals included in this study had taken antibiotics 5 years prior to sampling. Eight out of ten cancer patients were positive for $H$. pylori by one or more of four tests: culturing, immunohistochemistry, ELISA and immunoblotting (Enroth et al., 2000). However, only three of the patients (patients designated 2, 7 and 10) were positive for $H$. pylori by culturing alone. None of the controls were positive for H. pylori in any of the above-mentioned tests. Information about sociodemographic and clinical characteristics can be found in Table 1.

DNA isolation and PCR conditions. Biopsies from antrum and corpus were homogenized together and stored in a freezing buffer containing glycerol at $-70{ }^{\circ} \mathrm{C}$. One hundred microlitres of the homogenized suspension was transferred to a microcentrifuge tube containing $0.25 \mathrm{ml}$ of $0.1 \mathrm{~mm}$ zirconium beads with $180 \mu \mathrm{l}$ ATL lysis buffer solution (Qiagen). In addition, $20 \mu \mathrm{l}$ proteinase $\mathrm{K}$ was added to the tube. The bacterial cells in the samples were lysed by bead beating twice for $45 \mathrm{~s}$ at a setting of 5.0 using a FastPrep Instrument (MP Biomedicals). The DNA from the samples was then recovered using 
the QIAamp DNA Mini kit (Qiagen) according to the manufacturer's instructions for tissue samples, starting at step 3 in the protocol.

16S rRNA genes were amplified from the isolated DNA in three technical replicates per sample using the broad-range bacterial primers Bact 8F (5'-AGAGTTTGATCCTGGCTCAG-3'; Edwards et al., 1989), $5^{\prime}$-end-labelled with 6-carboxyfluorescein (6-FAM), and 926r (5'CCGTCAATTCCTTTRAGTTT-3'; Muyzer et al., 1993). Amplification was carried out in $25 \mu \mathrm{l}$ reaction mixtures under conditions that have been described previously (Dicksved et al., 2007), with the exception that the number of PCR cycles was increased to 35 instead of 30. PCRamplified DNA product amounts and sizes were confirmed by agarose gel electrophoresis using GeneRuler $100 \mathrm{bp}$ DNA ladder Plus (Fermentas Life Sciences) as a size marker.

T-RFLP. PCR products were digested with the HaelII restriction enzyme (GE Healthcare) and the digested fragments were separated on an ABI 3700 capillary sequencer, as previously described (Hjort et al., 2007). The sizes of the fluorescently labelled fragments were determined by comparison with the internal GS ROX-500 size standard [Applied Biosystems (ABI)]. T-RFLP electropherograms were imaged using GENESCAN software (ABI). Relative peak areas of each terminal restriction fragment (TRF) were determined by dividing the area of the peak of interest by the total area of peaks within the following threshold values: lower threshold at $50 \mathrm{bp}$ and upper threshold at $500 \mathrm{bp}$. A threshold for relative abundance was applied at $0.5 \%$ and only TRFs with higher relative abundances were included in the remaining analyses.

Cloning and sequencing. Cloning and sequencing of $16 \mathrm{~S}$ rRNA genes that were PCR-amplified from DNA extracted from the biopsy samples was performed to confirm the identities of bacterial species corresponding to the dominant TRFs. DNA samples from six cancer patients (nos 4, 5, 6, 7, 8 and 9) were selected for amplification with the general primers Bact $8 \mathrm{~F}$ and 926r. Three replicate PCR products from each individual were pooled and gel-purified using the Qiagen gel extraction kit. The pooled PCR products were cloned into the TOPO TA pCR 4.0 vector, followed by transformation into Escherichia coli TOP 10 competent cells (Invitrogen). A total of 64 inserts from each library were amplified using vector primers M13f and $\mathrm{M} 13 \mathrm{r}$ (Invitrogen) with the same thermal cycling program as for T-RFLP analysis. The PCR products were digested with HaeIII and HhaI (GE Healthcare) and digested fragments were separated in a $2.5 \%$ agarose gel. Band patterns, i.e. restriction fragment length polymorphism (RFLP) types, were analysed using GelCompare software (Applied Maths). Plasmids from all clones with unique RFLP types and several redundant RFLP types were purified and selected prior to sequence analysis; in total 140 clones. Obtained sequences were examined using MacVector 8.1.1 (Accelrys Software) to identify their corresponding TRF sizes and for removal of redundant sequences. The remaining sequences were aligned against GenBank database entries using standard nucleotide BLAST at NCBI (www.ncbi.nlm.nih.gov). Hits defined as unknown or uncultured bacteria were subsequently aligned against sequenced bacterial genomes (genomic BLAST at NCBI), as well as examined with the Ribosomal Database Project II Sequence Match, in an attempt to classify them. Sequences with 99-100 \% similarity were given the same name as the species hit whereas sequences with 97-99\% identity were assigned the genus name followed by 'sp.'. Sequences were aligned online using MAFFT aligner version 6 with the Q-INS-i strategy (Katoh \& Toh, 2008). A phylogenetic tree was constructed using PHYML v2.4.5 (Guindon \& Gascuel, 2003), and displayed using Figtree v1.0 (http://tree.bio.ed.ac.uk/software/figtree/), where large clusters were collapsed.

Statistical analysis. T-RFLP data from each individual were entered into a data matrix that consisted of the TRFs as variables and individuals as objects. A consensus T-RFLP profile was constructed for each of the technical duplicates. For significance testing of TRF distribution between the cancer patients and the controls, multiresponse permutation procedures were used with Bray-Curtis metrics as distances measure (PC-ORD; MjM Software). Cluster analysis was based on Bray-Curtis distances and a dendrogram was created using the software PAST (http://folk.uio.no/ohammer/past/). Diversity, defined as evenness and richness of the bacterial community members detected as TRFs by T-RFLP analysis, was calculated using Simpson's index of diversity $(D)$ (Begon et al., 2006). Differences in TRF abundances between groups were tested using the Mann-Whitney test and $P$-values $<0.05$ were considered statistically significant.

\section{RESULTS AND DISCUSSION}

The inhospitable acidic milieu in the stomach provides an effective barrier, killing many of the microbes that enter the gastrointestinal tract. However, a change of the physiological conditions of the stomach, as occurs during acidreducing drug therapy, corpus atrophy or gastric cancer, provides an opportunity for foreign microbes to enter and colonize the stomach. The evidence chain that targets $H$. pylori as a causative agent in gastric cancer development is long, but proof of the progression into cancer is lacking. Eradication of $H$. pylori was recently shown to decrease the incidence of gastric cancer (Fukase et al., 2008); however, the prophylactic effect of such eradication remains controversial (Malfertheiner et al., 2006). Duodenal ulcer disease, with overproduction of gastric acid, has been shown to protect against development of gastric cancer (Hansson et al., 1996). By contrast, several studies have suggested that an altered gastric physiology, as in corpus predominant gastritis, promotes bacterial overgrowth of the stomach and survival of potential genotoxic species with an unknown impact on human health (Calmels et al., 1987; Heavey \& Rowland, 2004). However, current knowledge of the identities of these microbial immigrants has been biased by the inability to cultivate a large fraction of the gut microbiota. Therefore, we used molecular approaches in this study to describe the gastric microbiota of patients suffering from gastric cancer.

\section{Analysis of clone libraries}

Clone libraries were generated from six cancer patients, and a total of 384 clones were screened for their individual RFLP types. Each RFLP type was expected to originate from similar target sequences. Therefore, to reduce the number of clones to sequence, all unique and redundant RFLP types were selected for sequencing. Out of the 140 clones sequenced, 102 phylotypes were found. These clustered into five bacterial phyla: Firmicutes, Bacteroidetes, Actinobacteria, Proteobacteria and Fusobacteria (Fig. 1). Members of the Firmicutes were most highly represented, with the majority corresponding to Streptococcus, Lactobacillus and different Clostridiales, such as Veillonella. The phylum Bacteroidetes was mainly represented by different species of Prevotella. All five classes of the Proteobacteria (Alpha-, Beta-, Gamma-, Delta- and Epsilonproteobacteria) were found in the clone libraries, with Neisseria and 


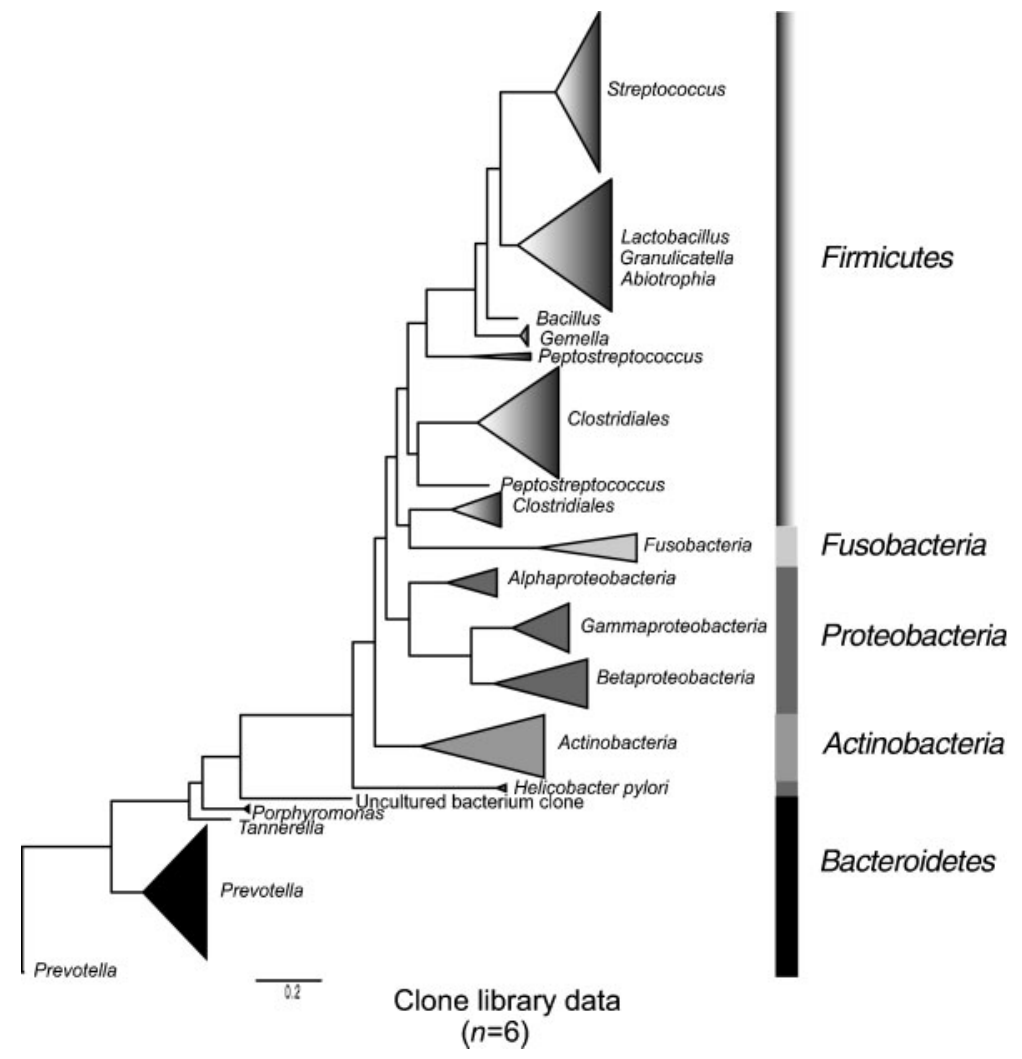

Fig. 1. Phylogenetic tree showing different phylotypes found in the stomach of six gastric cancer patients based on 16S rRNA gene sequences.

Haemophilus as the most dominant genera. H. pylori was only found in one of the libraries (no. 7), which was in concordance with previous culture-based results from this material (Table 1). In addition, members of the Actinobacteria and Fusobacteria were found, but clones corresponding to these phyla were not abundant.

\section{Correlation of clones to TRFs}

The TRF sizes of the sequenced clones were calculated in silico for their predicted lengths (base pairs) upon cleavage with the HaeIII enzyme. Positive correlations between sequenced clones and TRFs were judged based on the presence of both a TRF and a clone with the same TRF size (base pairs) in the same samples. Most of the TRFs could be correlated with the sequence data (Fig. 2). For many of the TRFs, only one genus matched with the particular fragment size, but a few TRFs had several genera that matched, e.g. TRFs 64, 205, 271 and 331. The most dominant TRFs, found in the majority of the individuals, were related to Streptococcus sp. (TRFs 307, 309 and 317), Prevotella (TRF 264), Veillonella (TRF 212) and different Proteobacteria (TRF 205) (Fig. 2). For some TRFs, mainly rare ones with abundances lower than $5 \%$, no matching sequences could be found in the libraries. In addition, some of the species found in the clone libraries had the restriction site outside the T-RFLP length-threshold values. This was the case for some members of the Proteobacteria, such as Klebsiella pneumoniae. By correlating TRFs to the sequence data, we found that the abundance of TRFs corresponding to Firmicutes represented $60 \%$ of the total population, followed by Bacteroidetes and Actinobacteria with relative abundances of $11 \%$ and $7 \%$, respectively (Fig. 3). TRFs that correlated with Proteobacteria and Fusobacteria were present in lower abundances: $6 \%$ and $3 \%$, respectively (Fig. 3). Approximately $12 \%$ of the T-RFLP abundance data could not be correlated with any sequence data (Fig. 3). This problem was mainly encountered for TRFs with low relative abundances, and many of the corresponding clones would probably have been found if more clones were sequenced. However, clones identified as Prevotella multiformis could be correlated with two different TRFs (177 and 264) possibly due to variations in the 16S rRNA gene sequences within this species.

\section{Analysis of the T-RFLP profiles}

The approach that we used in this study did not enable us to determine whether there were any differences in total abundances of bacteria between the cancer patients and the controls, but we could determine differences in relative abundances of some phyla. Analysis of the T-RFLP data revealed a total of 49 distinct TRFs and the mean number of TRFs found in an individual was 14 (range 7-20). None of the TRFs were present in all individuals and none were common for all cancer patients (Fig. 2). The diversity of 


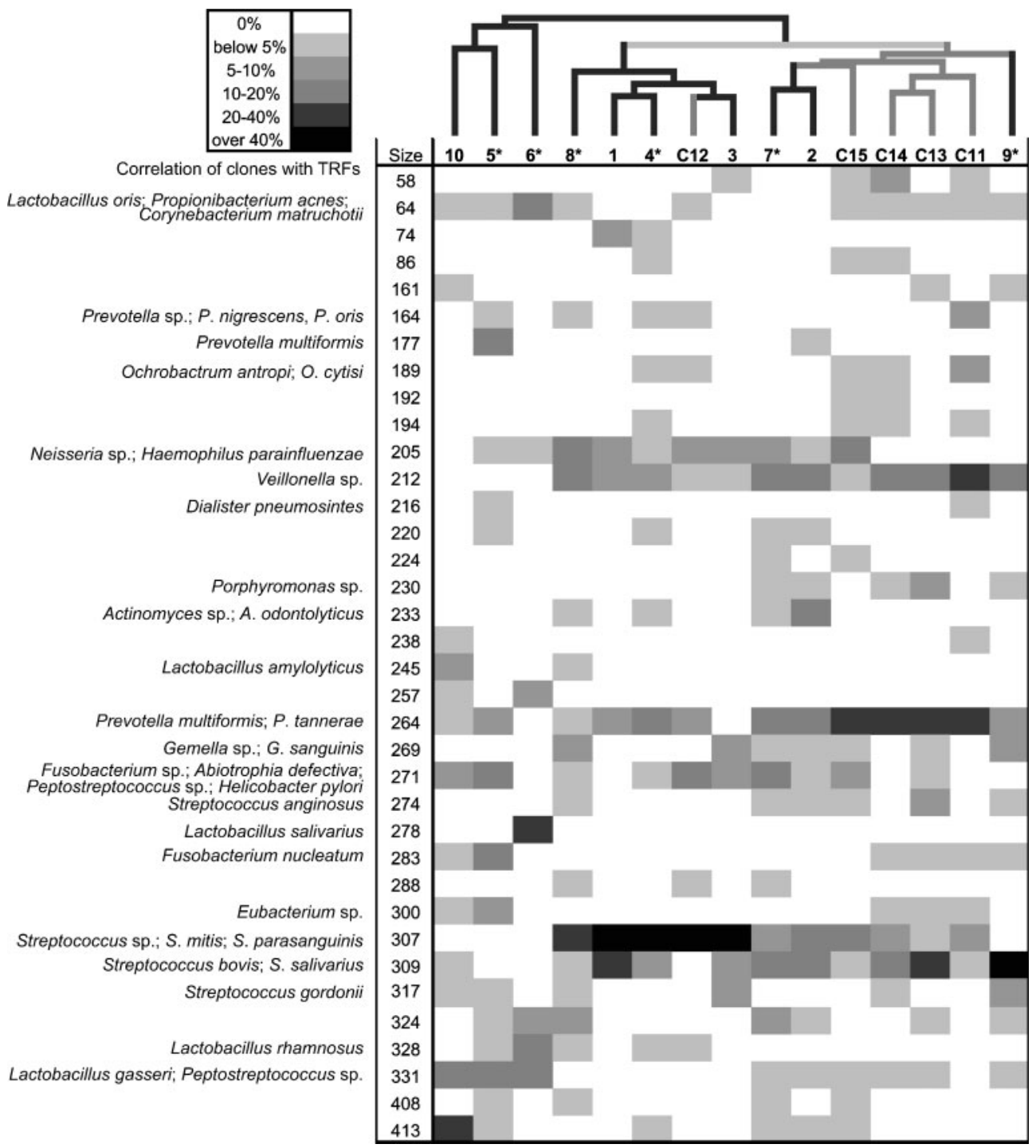

Fig. 2. Relative abundances of terminal restriction fragments (TRFs) corresponding to dominant bacterial populations in the stomach of gastric cancer patients and controls with a normal gastric mucosa. The explanation of the relative abundance intervals is shown in a progressive grey scale with higher abundances correlating with darker shades. The abundance profile for each sample is represented in a column and the abundance for each TRF in a row. To reduce space in the figure, TRFs that were only present in one of the samples and not correlated with sequence data were removed. The samples were sorted according to their clustering with the Bray-Curtis index as distance matrix and using the UPGMA method for the dendrogram. Branches in black represent gastric cancer patients and those in grey represent controls. *Samples that were selected for clone library construction and sequencing of the 16S rRNA genes (see Fig. 1). Sequences from the 16S rRNA gene clone library having matching TRF sizes when digested with Haell in silico and their closest BLAST hits are shown to the left of their corresponding TRF.

the microbiota was estimated based on the T-RFLP data using Simpson's index of diversity. There were no significant differences in diversity indices between the cancer patients and the control group. When the samples were clustered according to their TRF patterns, four out of five healthy controls clustered together (Fig. 3), suggesting that these bacterial communities are more similar to each other than to those of the cancer patients. In addition, three of the cancer patients differed significantly $(P=0.001)$ in their TRF distribution compared with the others (nos 5, 6 and 10; Fig. 2). In particular, the TRFs that corresponded to streptococci $(274,307,309$ and 317) were absent or very low in these three cancer patients. There was, however, no significant difference in the TRF distribution between all cancer patients and the healthy controls $(P=0.12)$ tested with multiresponse permutation procedures. 


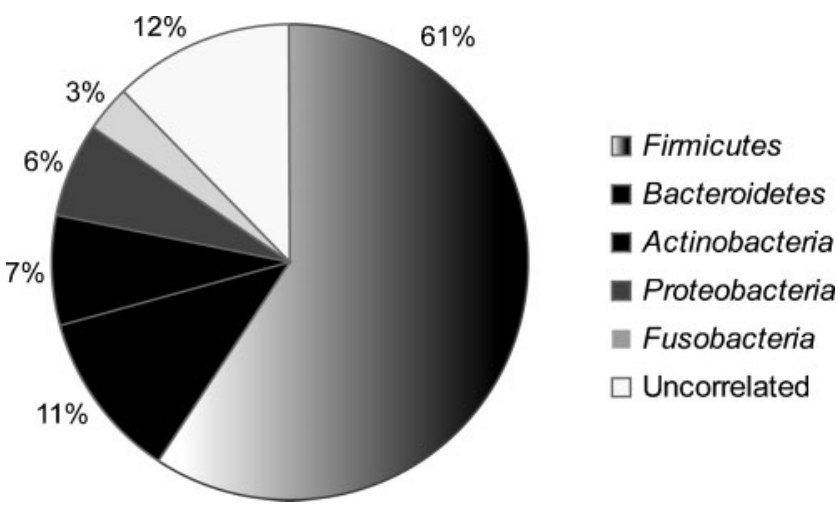

Fig. 3. Pie chart that shows the relative abundances of the different bacterial phyla found in six gastric cancer patients. The proportions of the different phyla are based on T-RFLP data, i.e abundance of TRFs that were correlated with sequence data. A grey scale represents different bacterial phyla according to the legend. TRFs with no matches in the clone libraries were classified as uncorrelated (white area).

\section{Abundance of $\boldsymbol{H}$. pylori}

The abundance of $H$. pylori in all samples included in this study was very low. The TRF (271) that corresponds to $H$. pylori was not among the most abundant ones and is not unique for $H$. pylori. The low abundance of $H$. pylori is in agreement with what has previously been reported in hypochlorhydric stomachs (Houben \& Stockbrügger, 1995). In a previous study, based on molecular approaches, $H$. pylori was detected in subjects that were considered negative based on traditional culturing or serological methods (Bik et al., 2006). In our study, clone libraries were created from six cancer patients and we only detected H. pylori in the one patient that was positive by culture, suggesting that $H$. pylori was absent in the other five individuals. However, only a limited number of clones were screened from each library and it is possible that we would have found $H$. pylori clones in these libraries by increasing the number of sequenced clones. Nevertheless, our results demonstrate a high microbial diversity in the stomach and are in accordance with a recent cloning survey of the stomach microbiota in hospitalized individuals (Bik et al., 2006), although the previous study did not include gastric cancer patients. The main difference in bacterial composition between this study and the study of Bik et al. (2006) was an overall lower prevalence of H. pylori and higher prevalence of clones or TRFs matching with lactobacilli in this study.

\section{Presence of Streptococcus bovis, a bacterium associated with colorectal cancer}

It has previously been shown that bacteria more frequently colonize gastric tumours compared to the surrounding mucosal tissues, and the most frequent colonizers of tumours are Gram-positive cocci such as streptococci (Sjöstedt et al., 1988). Streptococci are commonly isolated from the stomach and they have a wide repertoire of clinical features that can affect the host. For example, among the clones in this study, we found some that corresponded to $S$. bovis, a species that has been associated with colorectal cancer (Biarc et al., 2004; Ellmerich et al., 2000). It has been demonstrated that $S$. bovis or its antigens can promote cancer in rats, and that it exerted its pathological activity in the colonic mucosa only when pre-neoplastic lesions were established (Biarc et al., 2004). As for CagA-positive strains of $H$. pylori, S. bovis or its wall-extracted antigens can induce an overexpression of inflammatory mediators known to be associated with carcinogenesis, such as IL-8, prostaglandin $\mathrm{E}_{2}\left(\mathrm{PGE}_{2}\right)$ and cyclooxygenase (COX-2) (Jüttner et al., 2003). Further analysis of this group of bacteria is warranted to elucidate their potential involvement in gastric cancer progression.

\section{Acid-reducing therapy}

The control group in this study was hospital-based, and the individuals were relatively old, with a possible deficiency in acid production that may have influenced the results. Unfortunately, the $\mathrm{pH}$ of the gastric juice was not measured in the individuals partaking in this study. In hypochlorhydric stomachs, more bacteria may be in an actively growing state because of the more favourable $\mathrm{pH}$. This hypothesis is supported by previous investigations on persons that have taken acid-reducing drugs where a higher microbial diversity than normal was detected, and a correlation was found to species normally found in the oro-pharyngeal tract (Adamsson et al., 1999; Mowat et al., 2000). The microbial species composition found in the stomach during acid-reducing drug therapy shares many similarities with that found in the stomach of gastric cancer patients. This emphasizes the need for further research to characterize the structure and the activity of the microbiota in gastric cancer patients as well as in individuals undergoing acid-reducing drug therapy.

\section{$\mathbf{N}$-Nitroso compounds and gastric cancer}

Another factor that is thought to be a risk factor in developing gastric cancer is formation of N-nitroso compounds. Correa (1992) postulated that bacteria present in the hypochlorhydric stomach have the ability to reduce dietary nitrate to nitrite, and subsequently produce carcinogenic $\mathrm{N}$ nitroso compounds using secondary amines from food (Correa, 1992). It has also been reported that some of the species commonly found in the oral cavity have the ability to promote these reactions (Calmels et al., 1987; Ziebarth et al., 1997). In this study, several of the clones identified are normally found in the oral cavity, and may be potent promoters of this reaction, although this requires further investigation to confirm. 


\section{Conclusion}

In the current study, the gastric microbiota of patients with gastric cancer was characterized for the first time using molecular approaches and compared with that of dyspeptic controls having a normal healthy mucosa. Representatives of several genera were detected with a predominance of Firmicutes, mainly of oral origin. However, no significant differences in microbial compositions were found between cancer patients and controls. The prevalence of $H$. pylori was very low and this bacterium was only found in samples where it had previously been isolated using culture-based techniques. Although we only examined a limited number of subjects, this study contributes the first DNA-based description of the bacterial community composition in the stomachs of individuals with gastric cancer and shows the presence of a complex gastric microbiota with potential as a maintainer or bystander in the progression of gastric cancer.

\section{ACKNOWLEDGEMENTS}

This work was supported by the Uppsala BioX project, MICPROF, the Swedish Cancer Foundation, project 4518-B06-07, and in part by the US Department of Energy, Contract DE-AC02-05CH11231 with Lawrence Berkeley National Laboratory.

\section{REFERENCES}

Adamsson, I., Nord, C. E., Lundquist, P., Sjöstedt, S. \& Edlund, C. (1999). Comparative effects of omeprazole, amoxycillin plus metronidazole versus omeprazole, clarithromycin plus metronidazole on the oral, gastric and intestinal microflora in Helicobacter pyloriinfected patients. J Antimicrob Chemother 44, 629-640.

Andersson, A. F., Lindberg, M., Jakobsson, H., Bäckhed, F., Nyren, P. \& Engstrand, L. (2008). Comparative analysis of human gut microbiota by barcoded pyrosequencing. PLoS One 3, e2836.

Begon, M., Harper, J. L. \& Townsend, C. R. (editors) (2006). The nature of the community, patterns in space and time. In Ecology: from Individuals to Ecosystems, pp. 471-472. Oxford: Blackwell.

Biarc, J., Nguyen, I. S., Pini, A., Gossè, F., Richert, S., Thiersé, D., Van Dorsselaer, A., Leize-Wagner, E., Raul, F. \& other authors (2004). Carcinogenic properties of proteins with pro-inflammatory activity from Streptococcus infantarius (formerly S. bovis). Carcinogenesis 25, 1477-1484.

Bik, E. M., Eckburg, P. B., Gill, S. R., Nelson, K. E., Purdom, E. A., Francois, F., Perez-Perez, G., Blaser, M. J. \& Relman, D. A. (2006). Molecular analysis of the bacterial microbiota in the human stomach. Proc Natl Acad Sci U S A 103, 732-737.

Blaser, M. J. \& Atherton, J. C. (2004). Helicobacter pylori persistence: biology and disease. J Clin Invest 113, 321-333.

Calmels, S., Ohshima, H., Crespi, M., Leclerc, H., Cattoen, C. \& Bartsch, H. (1987). N-nitrosamine formation by microorganisms isolated from human gastric juice and urine: biochemical studies on bacteria-catalysed nitrosation. IARC Sci Publ 105, 391-395.

Correa, P. (1992). Human gastric carcinogenesis: a multistep and multifactorial process - First American Cancer Society Award Lecture on Cancer Epidemiology and Prevention. Cancer Res 52, 6735-6740.

Dicksved, J., Flöistrup, H., Bergström, A., Rosenquist, M., Pershagen, G., Scheynius, A., Roos, S., Alm, J. S., Engstrand, L. \& other authors (2007). Molecular fingerprinting of the fecal microbiota of children raised according to different lifestyles. Appl Environ Microbiol 73, 2284-2289.

Eckburg, P. B., Bik, E. M., Bernstein, C. N., Purdom, E., Dethlefsen, L., Sargent, M., Gill, S. R., Nelson, K. E. \& Relman, D. A. (2005). Diversity of the human intestinal microbial flora. Science 308, 1635-1638.

Edwards, U., Rogall, T., Blöcker, H., Emde, M. \& Böttger, E. C. (1989). Isolation and direct complete nucleotide determination of entire genes - characterization of a gene coding for 16S-ribosomal RNA. Nucleic Acids Res 17, 7843-7853.

Ellmerich, S., Schöller, M., Duranton, B., Gosse, F., Galluser, M., Klein, J. P. \& Raul, F. (2000). Promotion of intestinal carcinogenesis by Streptococcus bovis. Carcinogenesis 21, 753-756.

Enroth, H., Kraaz, W., Engstrand, L., Nyren, O. \& Rohan, T. (2000). Helicobacter pylori strain types and risk of gastric cancer: a casecontrol study. Cancer Epidemiol Biomarkers Prev 9, 981-985.

Fukase, K., Kato, M., Kikuchi, S., Ozawa, K., Minoura, T., Konno, M., Maisawa, S., Toyoda, S., Yoshimura, N. \& other authors (2008). Effect of eradication of Helicobacter pylori on incidence of metachronous gastric carcinoma after endoscopic resection of early gastric cancer: an open-label, randomised controlled trial. Lancet 372, 392-397.

Guindon, S. \& Gascuel, O. (2003). A simple, fast, and accurate algorithm to estimate large phylogenies by maximum likelihood. Syst Biol 52, 696-704.

Hansson, L. E., Nyren, O., Hsing, A. W., Bergström, R., Josefsson, S., Chow, W. H., Fraumeni, J. F., Jr \& Adami, H. O. (1996). The risk of stomach cancer in patients with gastric or duodenal ulcer disease. $N$ Engl J Med 335, 242-249.

Heavey, P. M. \& Rowland, I. R. (2004). Microbial-gut interactions in health and disease. Gastrointestinal cancer. Best Pract Res Clin Gastroenterol 18, 323-336.

Hjort, K., Lembke, A., Speksnijder, A., Smalla, K. \& Jansson, J. K. (2007). Community structure of actively growing bacterial populations in plant pathogen suppressive soil. Microb Ecol 53, 399-413.

Houben, G. M. \& Stockbrügger, R. W. (1995). Bacteria in the aetiopathogenesis of gastric cancer: a review. Scand J Gastroenterol Suppl 212, 13-18.

Jüttner, S., Cramer, T., Wessler, S., Walduck, A., Gao, F., Schmitz, F., Wunder, C., Weber, M., Fischer, S. M. \& other authors (2003). Helicobacter pylori stimulates host cyclooxygenase-2 gene transcription: critical importance of MEK/ERK-dependent activation of USF1/-2 and CREB transcription factors. Cell Microbiol 5, 821-834.

Kato, S., Nakajima, S., Nishino, Y., Ozawa, K., Minoura, T., Konno, M., Maisawa, S., Toyoda, S., Yoshimura, N. \& other authors (2006). Association between gastric atrophy and Helicobacter pylori infection in Japanese children: a retrospective multicenter study. Dig Dis Sci 51, 99-104.

Katoh, K. \& Toh, H. (2008). Recent developments in the MAFFT multiple sequence alignment program. Brief Bioinform 9, 286-298.

Malfertheiner, P., Fry, L. C. \& Monkemuller, K. (2006). Can gastric cancer be prevented by Helicobacter pylori eradication? Best Pract Res Clin Gastroenterol 20, 709-719.

Monstein, H. J., Tiveljung, A., Kraft, C. H., Borch, K. \& Jonasson, J. (2000). Profiling of bacterial flora in gastric biopsies from patients with Helicobacter pylori-associated gastritis and histologically normal control individuals by temperature gradient gel electrophoresis and $16 \mathrm{~S}$ rDNA sequence analysis. J Med Microbiol 49, 817-822.

Mowat, C., Williams, C., Gillen, D., Hossack, M., Gilmour, D., Carswell, A., Wirz, A., Preston, T. \& McColl, K. E. (2000). Omeprazole, Helicobacter pylori status, and alterations in the intragastric milieu facilitating bacterial N-nitrosation. Gastroenterology 119, 339-347. 
Muyzer, G., Dewaal, E. C. \& Uitterlinden, A. G. (1993). Profiling of complex microbial populations by denaturing gradient gel electrophoresis analysis of polymerase chain reaction amplified genes coding for 16S ribosomal-RNA. Appl Environ Microbiol 59, 695-700.

Schorah, C. J., Sobala, G. M., Sanderson, M., Collis, N. \& Primrose, J. N. (1991). Gastric juice ascorbic acid: effects of disease and implications for gastric carcinogenesis. Am J Clin Nutr 53, 287S-293S.

Sjöstedt, S., Kager, L., Heimdahl, A. \& Nord, C. E. (1988). Microbial colonization of tumors in relation to the upper gastrointestinal tract in patients with gastric carcinoma. Ann Surg 207, 341-346.
Smith, M. G., Hold, G. L., Tahara, E. \& El-Omar, E. M. (2006). Cellular and molecular aspects of gastric cancer. World J Gastroenterol 12, 2979-2990.

Uemura, N., Okamoto, S., Yamamoto, S., Matsumura, N., Yamaguchi, S., Yamakido, M., Taniyama, K., Sasaki, N. \& Schlemper, R. J. (2001). Helicobacter pylori infection and the development of gastric cancer. $N$ Engl J Med 345, 784-789.

Ziebarth, D., Spiegelhalder, B. \& Bartsch, H. (1997). N-nitrosation of medicinal drugs catalysed by bacteria from human saliva and gastrointestinal tract, including Helicobacter pylori. Carcinogenesis 18, 383-389. 